\title{
Acase report of sporadic Creutzfeldt-Jakob disease in an Asian origin coronavirus disease-19 patient: An enigma
}

\author{
Suman Choudhary ${ }^{1}$, Mayank Sonkar ${ }^{2}$, Ashok Kumar Saxena ${ }^{3}$ \\ From ${ }^{1}$ Senior Resident, ${ }^{2}$ Post Graduate Student, Department of Anesthesiology, University College of Medical Sciences College, ${ }^{3}$ Head and Professor, \\ Department of Anesthesiology and Critical Care, Guru Teg Bahadur Hospital and University College of Medical Sciences College, New Delhi, Delhi, \\ India
}

\begin{abstract}
Creutzfeldt-Jakob disease (CJD) is a rare, degenerative, and fatal brain disorder affecting about one in every 1 million persons/year worldwide, with a typical duration from onset to death is only 7 months. In our case report, we describe a patient of Sporadic CJD (sCJD) who presented to the hospital during the coronavirus disease (COVID-19) pandemic and the intensive care unit experience and precautions taken to prevent the iatrogenic spread of the disease. This case was diagnosed following confirmation of all the typical clinical, radiological, and laboratory features of this condition. From all recent data on the pathogenesis of the sub-category of prion disease and the immune responses to severe acute respiratory syndrome coronavirus-2, we can suggest that coronavirus disease can lead to exaggerated and accelerated pathogenesis and manifestations of neurodegenerative disorders such as of our patient's sCJD. We have tried to highlight the challenges in managing patients with a rare disease such as $\mathrm{SCJD}$ and how its progression can be intensified by COVID-19.
\end{abstract}

Key words: Brain, Coronavirus disease 19, Neurodegenerative disorder, Sporadic Creutzfeldt-Jakob disease

$\mathrm{C}$ reutzfeldt-Jakob disease (CJD) is a rare, degenerative, fatal brain disorder affecting about one in every 1 million persons/year worldwide [1]. It is the prototype of a family of rare and fatal human degenerative conditions characterized by progressive brain dysfunction. CJD falls into four categories: sporadic, familial, iatrogenic, and variant. Sporadic CJD (sCJD), thought to occur worldwide, is the most common form and represents about $85 \%$ of all CJD cases; the incidence ranges between 0.5 and 1.5 cases per million inhabitants per year [2]. An early diagnosis is important for counseling the patient and their family members, as also for optimal management of symptoms and to avoid any iatrogenic transmission. However, any delay in diagnosing the disease is due to a combination of its rarity as well as heterogeneity in the early stages of the disease [3].

We report our coronavirus disease (COVID-19) intensive care unit experience in a patient of SCJD who presented with respiratory symptoms and seizures, which were different from the typical neuropsychiatric presentation of sCJD. Hence, we describe the challenges faced in diagnosis, management, and precautionary measurements taken for such a patient, whose

\section{Access this article online}

Received- 05 April 2021

Initial Review- 22 April 2021

Accepted- 03 May 2021

DOI: $10.32677 /$ IJCR.2021.v07.i05.005 condition got aggravated and worsened by the concurrent COVID-19.

\section{CASE REPORT}

A 61-year-old female presented with symptoms of heaviness over the base of the head which progressively worsened over 2 months, along with difficulty in walking, "tremulousness" and low-grade fever on and off. Gradually, she developed a few episodes of confusion with irrelevant and abnormal behavior which worsened over a week along with visual hallucinations.

The clinical presentation of the patient suggested some neurodegenerative disease for which, she was referred to the Institute of Human Behavior and Allied Sciences, Delhi, India, where the diagnosis of CJD was made based on the clinical symptoms, typical electroencephalograph (EEG), and magnetic resonance imaging $(\mathrm{MRI})$.

The patient was admitted for the above complaints, where she was clinically suspected to be a case of autoimmune encephalitis. Contrast-enhanced MRI was done which revealed empty "sella turcica," and she was treated with methylprednisolone and intravenous immune globulin. The patient's condition showed little improvement for a while and later started to develop

Correspondence to: Dr. Suman Choudhary, 377, Kamala Nehru Nagar, Chopasani Road, Jodhpur - 342 001, Rajasthan, India. E-mail: choudharydrsuman@gmail.com

(C) 2021 Creative Commons Attribution-NonCommercial 4.0 International License (CC BY-NC-ND 4.0). 
myoclonus and rigidity. Her condition further worsened and she started having difficulty in movement and initiation (freezing) and even had difficulty in carrying out her day-to-day activities, gradually leading to a bedridden status.

Series of contrast enhanced-MRI and EEG were done which revealed hyperintense signals in basal ganglia (Fig. 1a) and thalamus (Fig. 1b) with "cortical ribboning" (Fig. 1c) and $1 \mathrm{~Hz}$ periodic wave discharges on EEG. Laboratory tests of paraneoplastic neuronal antibody serum, anti-thyroid peroxidase, microsomal antibody, neuron-specific enolase, etc., were done and by the criteria of exclusion, she was diagnosed as a case of SCJD. We planned for the brain biopsy for confirmation of CJD, but it was not possible for the patient due to COVID-19. Chest X-ray was done for fever and respiratory distress, which was suggestive of bilateral pneumonitis. Her reverse transcriptase-polymerase chain reaction (RT-PCR) was positive for severe acute respiratory syndrome coronavirus- 2 and so, she was referred back to our COVID-19 dedicated hospital for further management.

At the time of admission in ICU, she was in respiratory distress along with fever with $\mathrm{SpO}_{2}$ of only $78-80 \%$ on room air, which improved to $98-100 \%$ after oxygen therapy through a non-rebreather mask at $15 \mathrm{l} / \mathrm{min}$. The mental status of the patient ranged from stupor to semi-coma and her vital parameters were stable with a blood pressure of $138 / 82 \mathrm{~mm}$ of $\mathrm{Hg}$ and a heart rate of $82 \mathrm{bpm}$, regular. Later on, the patient developed seizures which were managed symptomatically with benzodiazepines and sodium valproate, following which she developed profound hypotension and bradycardia, which was managed with noradrenaline infusion.

Being a COVID-19 dedicated hospital with all standard precautionary measures, the patient was shifted to isolation cubical where staff in contact was minimized. A huge challenge with SCJD is that the small size of prions facilitates them to be localized in the minute clefts on equipment and is supposed to be resistant to various sterilization techniques. Hence, keeping this in mind, all the disposable circuits, facemask, and filters were disposed of separately with special attention to the IV cannula, central line, and Foley's catheter.

A week later, a repeat RT-PCR test was done and for which our patient tested negative. The patient stayed in ICU, bedridden, with oxygen support as she was unable to maintain her $\mathrm{SpO}_{2}$ levels in room air. Over a period of a month and despite our best efforts incorporating a multimodal approach consisting of remdesivir 100 $\mathrm{mg}$, convalescent plasma therapy, Methylprednisolone $62.5 \mathrm{mg}$, and low molecular weight heparin (LMWH) $60 \mathrm{mg}(0.6 \mathrm{ml})$ along with REGULAR physical therapy, dietary and nutritional supplements, and standard ICU care as per the standard protocol, which led to some improvement as shown in Table 1., this patient could not survive past 12 weeks from her initial onset of symptoms.

\section{DISCUSSION}

In this case report of COVID-19 positive SCJD afflicted 61-year-old female nursing officer, we realized that already there are so many reported cases of CJD published in peer-reviewed journals of the pre-pandemic era, whereas, in the last 7 months, there have been only two case reports of CJD with one being RT-PCR positive and another without any laboratory evidence. The current case report happens to be the very first Asian origin SCJD patient who tested positive for COVID-19 on RTPCR 5 $\frac{1}{2}$ weeks before her death.

The current case report of COVID-19 positive CJD's had typical COVID-19 manifestations of respiratory symptoms such as tachypnea, respiratory distress, cough, and fever; whereas, the other two reported cases of SCJD with COVID-19 did not have any respiratory symptoms whatsoever $[4,5]$. With specific reference to our case report, the patient experienced repeated generalized tonic-clonic seizure during her prolonged stay in COVID-19 ICU. As regards to our case report, this patient experienced typical autonomic dysfunction in the form of dominant parasympathetic system activity such as bradycardia, whereas other reported cases did not exhibit seizure activity. Those two cases report never had seizures [4,5], but our patient had to be managed with levetiracetam, sodium valproate, and phenytoin.

Again, with specific reference to our case report, our patient showed severe anemia of hemoglobin $5 \mathrm{~g} / \mathrm{dl}$, whereas other case reports did not show evidence of low hemoglobin $[4,5]$. There is so much variability in the survival period among SCJD patients that in our case, it was 12 weeks, whereas, in Young et al., it was 4 weeks [4]. From recent literature on association with Prion disease and COVID-19, a hypothesis has been postulated that the cascade of systemic inflammation mediators in response to the virus accelerated the pathogenesis and hastened the preclinical and neurodegenerative disorder.

Recently Young et al. [4] concluded in their case report with the comment that there is bound to be a possible sudden surge in the systemic inflammatory response to viral load due to COVID19. In another case report by McMurran et al., the authors also
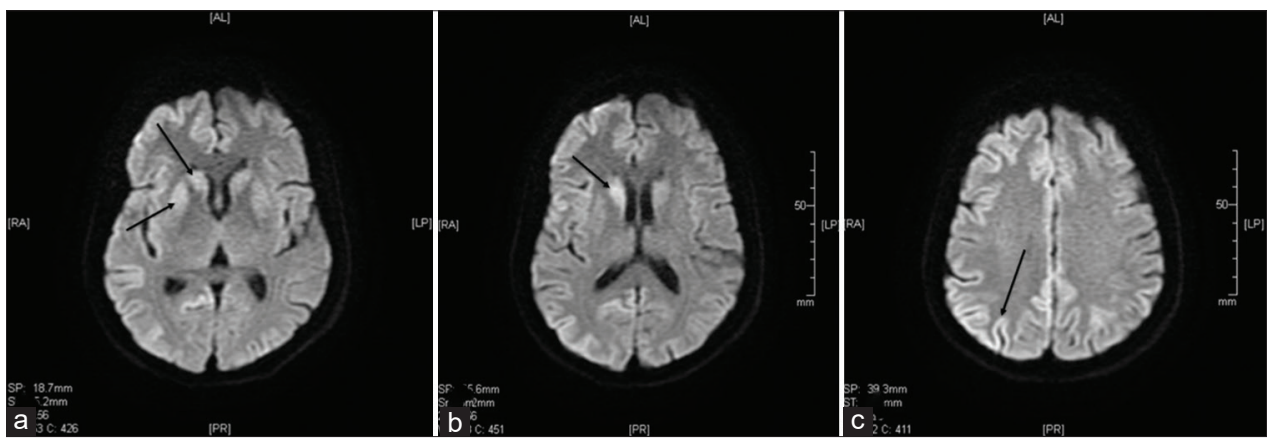

Figure 1: Hyperintense signals observed in the (a) basal ganglia (caudate and putamen) and (b) caudate nucleus of this patient diagnosed as SCJD (c)“Cortical ribboning" observed in this patient diagnosed as SCJD 
Table 1: Trend of systemic inflammatory markers in this patient

\begin{tabular}{lcccc}
\hline Investigations & $\begin{array}{c}\text { On } \\
\text { admission }\end{array}$ & & $\begin{array}{c}\text { After } \\
\text { treatment } \\
\text { (Day 20 of } \\
\text { admission) }\end{array}$ & Ref. range \\
\hline D-dimer & $1530 \mathrm{ng} / \mathrm{ml}$ & $->$ & $1360 \mathrm{ng} / \mathrm{ml}$ & $220-740 \mathrm{ng} / \mathrm{ml}$ \\
PCT & $0.22 \mathrm{ng} / \mathrm{ml}$ & $->$ & $0.16 \mathrm{~g} / \mathrm{ml}$ & $<0.15 \mathrm{ng} / \mathrm{ml}$ \\
IL-6 & $21.7 \mathrm{pg} / \mathrm{ml}$ & $->$ & $5.2 \mathrm{pg} / \mathrm{ml}$ & $0.00-7.00 \mathrm{pg} / \mathrm{ml}$ \\
Ferritin & $>1650 \mathrm{ng} / \mathrm{ml}$ & $->$ & $1193 \mathrm{ng} / \mathrm{ml}$ & $45-204 \mathrm{ng} / \mathrm{ml}$ \\
Troponin-T & $0.072 \mathrm{ng} / \mathrm{ml}$ & $->$ & $0.000 \mathrm{ng} / \mathrm{ml}$ & $<0.04 \mathrm{ng} / \mathrm{ml}$ \\
\hline
\end{tabular}

concluded with the same hypothesis of accelerated progression of CJD [5]. Similarly, it reflects that in this case report, there may be a sudden surge in systemic inflammatory mediators due to COVID infection, which might have led to the early demise of the patient despite our best efforts incorporating a multimodal approach consisting of remdesivir $100 \mathrm{mg}$, convalescent plasma therapy, methylprednisolone $62.5 \mathrm{mg}$, and LMWH $60 \mathrm{mg}(0.6 \mathrm{ml})$ along with regular physical therapy, dietary, nutritional supplements, and standard ICU care as per the standard protocol [6-8].

In such conditions, all necessary measures should be taken to substantially minimize the possibility of transmission between CJD patient and hospital worker and the number of staff in contact to be kept in minimal. Farling et al. in a practical guide reported localization of small prions in equipment's microscopic crypts and their resistant nature to all sterilization techniques, including high dose ionizing radiation, ultraviolet radiation, and autoclaving [7]. In sCJD, along with COVID-19, we must advise remdesivir, plasma therapy, methylprednisolone, and LMWH. This patient remained comfortable and stable in the COVID-19 ICU all throughout her stay and till the time of her death.

\section{CONCLUSION}

To conclude, when a patient of SCJD gets infected with COVID-19, the neurological deterioration gets exacerbated and overall survival shortens and hence, there is a need for extra-vigilance and intensive monitoring for these patients. Intensivists and anesthesiologists should be aware of the nature of SCJD and precautions to be taken. An expeditious diagnosis, swift recognition, management, and precautions are of utmost importance, simply because this disease is notably associated with a high degree of morbidity and mortality.

\section{REFERENCES}

1. Uttley L, Carroll C, Wong R, Hilton DA, Stevenson M. Creutzfeldt-Jakob disease: A systematic review of global incidence, prevalence, infectivity, and incubation. Lancet Infect Dis 2020;20:e2-10.

2. Manix M, Kalakoti P, Henry M, Thakur J, Menger R, Guthikonda B, et al. Creutzfeldt-Jakob disease: Updated diagnostic criteria, treatment algorithm, and the utility of brain biopsy. Neurosurg Focus 2015;39:E2.

3. Carswell C, Thompson A, Lukic A, Stevens J, Rudge P, Mead S, et al. MRI findings are often missed in the diagnosis of Creutzfeldt-Jakob disease. BMC Neurol 2012;12:153.

4. Young MJ, O'Hare M, Matiello M, Schmahmann JD. CreutzfeldtJakob disease in a man with COVID-19: SARS-CoV-2-accelerated neurodegeneration? Brain Behav Immun 2020;89:601-3.

5. McMurran CE, Chaggar GH, Ugoya SO. A patient with sporadic CreutzfeldtJakob disease: Challenges of rare diseases in the COVID-19 era. Oxf Med Case Reports 2020;2020:omaa113.

6. Siordia JA Jr., Bernaba M, Yoshino K, Ulhaque A, Kumar S, Bernaba M, et al. Systematic and statistical review of Coronavirus disease 19 treatment trials. SN Compr Clin Med 2020;Jul 15:1-12.

7. In CB, Choi YS, Park EY. Anesthetic management in patients suspected of Creutzfeldt-Jakob disease-a case report. Korean J Anesthesiol 2011;61:262-4

8. Leisman DE, Deutschman CS, Legrand M. Facing COVID-19 in the ICU: Vascular dysfunction, thrombosis, and dysregulated inflammation. Intensive Care Med 2020;46:1105-8

Funding: None; Conflicts of Interest: None Stated.

How to cite this article: Choudhary S, Sonkar M, Saxena AK. A case report of sporadic Creutzfeldt-Jakob disease in an Asian origin coronavirus disease-19 patient: An enigma. Indian J Case Reports. 2021;7(5):188-190. 